\title{
Every maximally monotone operator of Fitzpatrick-Phelps type is actually of dense type
}

\author{
Heinz H. Bauschke, Jonathan M. Borwein† Xianfu Wang
}

April 4, 2011

\begin{abstract}
We show that every maximally monotone operator of Fitzpatrick-Phelps type defined on a real Banach space must be of dense type. This provides an affirmative answer to a question posed by Stephen Simons in 2001 and implies that various important notions of monotonicity coincide.
\end{abstract}

\section{Mathematics Subject Classification:}

Primary 47H05; Secondary 46B10, 47N10, 90C25

Keywords: Fitzpatrick function, maximally monotone operator, monotone operator, multifunction, operator of type (D), operator of type (FP), operator of type (NI), set-valued operator.

\section{Introduction}

Throughout this note, we assume that $X$ is a real Banach space with norm $\|\cdot\|$, that $X^{*}$ is the continuous dual of $X$, and that $X$ and $X^{*}$ are paired by $\langle\cdot, \cdot\rangle$. Let $A: X \rightrightarrows X^{*}$ be

*Mathematics, Irving K. Barber School, University of British Columbia, Kelowna, B.C. V1V 1V7, Canada. E-mail: heinz.bauschke@ubc.ca.

†CARMA, University of Newcastle, Newcastle, New South Wales 2308, Australia. E-mail: jonathan. borwein@newcastle.edu. au.

${ }^{\ddagger}$ Mathematics, Irving K. Barber School, University of British Columbia, Kelowna, B.C. V1V 1V7, Canada. E-mail: shawn.wang@ubc.ca.

$\S$ Mathematics, Irving K. Barber School, University of British Columbia, Kelowna, B.C. V1V 1V7, Canada. E-mail: 1jinyao@interchange.ubc.ca. 
a set-valued operator (also known as multifunction) from $X$ to $X^{*}$, i.e., for every $x \in X$, $A x \subseteq X^{*}$, and let gra $A=\left\{\left(x, x^{*}\right) \in X \times X^{*} \mid x^{*} \in A x\right\}$ denote the graph of $A$. The domain of $A$ is $\operatorname{dom} A=\{x \in X \mid A x \neq \varnothing\}$, while ran $A=A(X)$ is the range of $A$. Recall that $A$ is monotone if

$$
\left\langle x-y, x^{*}-y^{*}\right\rangle \geq 0, \quad \forall\left(x, x^{*}\right) \in \operatorname{gra} A \forall\left(y, y^{*}\right) \in \operatorname{gra} A,
$$

and maximally monotone if $A$ is monotone and $A$ admits no proper monotone extension. It will be convenient to also say that gra $A$ is monotone or maximally monotone respectively in this case. We can then simply say that $\left(x, x^{*}\right) \in X \times X^{*}$ is monotonically related to gra $A$ if $\left\{\left(x, x^{*}\right)\right\} \cup \operatorname{gra} A$ is monotone.

We now recall the three fundamental types of monotonicity.

Definition 1.1 Let $A: X \rightrightarrows X^{*}$ be maximally monotone. Then three key types of monotone operators are defined as follows.

(i) $A$ is of dense type or type (D) (1971, [11]) if for every $\left(x^{* *}, x^{*}\right) \in X^{* *} \times X^{*}$ with

$$
\inf _{\left(a, a^{*}\right) \in \operatorname{gra} A}\left\langle a-x^{* *}, a^{*}-x^{*}\right\rangle \geq 0,
$$

there exist a bounded net $\left(a_{\alpha}, a_{\alpha}^{*}\right)_{\alpha \in \Gamma}$ in gra $A$ such that $\left(a_{\alpha}, a_{\alpha}^{*}\right)_{\alpha \in \Gamma}$ weak ${ }^{*} \times$ strong converges to $\left(x^{* *}, x^{*}\right)$.

(ii) $A$ is of type negative infimum (NI) (1996, [16]) if

$$
\sup _{\left(a, a^{*}\right) \in \operatorname{gra} A}\left(\left\langle a, x^{*}\right\rangle+\left\langle a^{*}, x^{* *}\right\rangle-\left\langle a, a^{*}\right\rangle\right) \geq\left\langle x^{* *}, x^{*}\right\rangle, \quad \forall\left(x^{* *}, x^{*}\right) \in X^{* *} \times X^{*} .
$$

(iii) $A$ is of type Fitzpatrick-Phelps (FP) (1992, [10]) if whenever $U$ is an open convex subset of $X^{*}$ such that $U \cap \operatorname{ran} A \neq \varnothing, x^{*} \in U$, and $\left(x, x^{*}\right) \in X \times X^{*}$ is monotonically related to gra $A \cap(X \times U)$ it must follow that $\left(x, x^{*}\right) \in \operatorname{gra} A$.

All three of these properties are known to hold for the subgradient of a closed convex function and for every maximally monotone operator on a reflexive space. These and other relationships known amongst these and other monotonicity notions are described in [6. Chapter 8]. Monotone operators are fundamental objects in modern Optimization and Variational Analysis; see, e.g., [3, 4, 5], the books [2, 6, 7, 14, 17, 19, 15, 21] and the references therein.

In Theorem 3.1 of this paper, we provide an affirmative to the following question, posed by S. Simons [18, Problem 18, page 406]:

Let $A: X \rightrightarrows X^{*}$ be maximally monotone such that $A$ is of type (FP). Is A necessarily of type (D)? 
In consequence, in Corollary 3.2 we record that the three notions in Definition 1.1 actually coincide.

We shall utilize the following notation, in addition to standard notions from convex analysis: The open unit ball in $X$ is $U_{X}=\{x \in X \mid\|x\|<1\}$, and the closed unit ball is $\{x \in X \mid\|x\| \leq 1\}$. It is very convenient to identify $X$ with its canonical image in the bidual space $X^{* *}$. Moreover, $X \times X^{*}$ and $\left(X \times X^{*}\right)^{*}=X^{*} \times X^{* *}$ are paired via

$$
\left\langle\left(x, x^{*}\right),\left(y^{*}, y^{* *}\right)\right\rangle=\left\langle x, y^{*}\right\rangle+\left\langle x^{*}, y^{* *}\right\rangle,
$$

where $\left(x, x^{*}\right) \in X \times X^{*}$ and $\left(y^{*}, y^{* *}\right) \in X^{*} \times X^{* *}$. We recall the following basic fact regarding the second dual ball:

Fact 1.2 (Goldstine) (See [13, Theorem 2.6.26] or [8, Theorem 3.27].) The weak*-closure of $B_{X}$ in $X^{* *}$ is $B_{X^{* *}}$.

The remainder of this paper is organized as follows. In Section 2, we record auxiliary results for subsequent use. The main result (Theorem [3.1) and the promised corollary (Corollary 3.2) are provided in Section 3 .

\section{Preliminary monotonicity results}

A now fundamental tool of modern monotone operator theory originated with Simon Fitzpatrick in 1988. It is reprised next:

Fact 2.1 (Fitzpatrick) (See [9, Corollary 3.9].) Let $A$ : $X \rightrightarrows X^{*}$ be maximally monotone, and let us set

$$
\left.\left.F_{A}: X^{* *} \times X^{*} \rightarrow\right]-\infty,+\infty\right]:\left(x^{* *}, x^{*}\right) \mapsto \sup _{\left(a, a^{*}\right) \in \operatorname{gra} A}\left(\left\langle x^{* *}, a^{*}\right\rangle+\left\langle a, x^{*}\right\rangle-\left\langle a, a^{*}\right\rangle\right) .
$$

Then for every $\left(x, x^{*}\right) \in X \times X^{*}$, the inequality

$$
\left\langle x, x^{*}\right\rangle \leq F_{A}\left(x, x^{*}\right)
$$

is true, and equality holds if and only if $\left(x, x^{*}\right) \in \operatorname{gra} A$.

The function $\left.F_{A}\right|_{X \times X^{*}}$ is the classical Fitzpatrick function associated with $A$.

The first relevant relationship established for (FP) operators is due to Stephen Simons:

Fact 2.2 (Simons) (See [18, Theorem 17] or [19, Theorem 37.1].) Let $A: X \rightrightarrows X^{*}$ be maximally monotone and of type (D). Then $A$ is of type (FP). 
The most powerful current information is captured in the following result.

Fact 2.3 (Simons / Marques Alves and Svaiter) (See [16, Lemma 15] or [19, Theorem 36.3(a)], and [12, Theorem 4.4].) Let $A: X \rightrightarrows X^{*}$ be maximally monotone. Then $A$ is of type (D) if and only if it is of type (NI).

The implication type (NI) implies type (D) — which we exploit — is very recently due to Marques Alves and Svaiter [12].

\section{Main result}

The next theorem is our main result. In conjunction with the corollary that follows, it provides the affirmative answer promised to Simons's problem posed in [18, Problem 18].

Theorem 3.1 Let $A: X \rightrightarrows X^{*}$ be maximally monotone such that $A$ is of type (FP). Then $A$ is of type (NI).

Proof. After translating the graph if necessary, we can and do suppose that $(0,0) \in \operatorname{gra} A$. Let $\left(x_{0}^{* *}, x_{0}^{*}\right) \in X^{* *} \times X^{*}$. We must show that

$$
F_{A}\left(x_{0}^{* *}, x_{0}^{*}\right) \geq\left\langle x_{0}^{* *}, x_{0}^{*}\right\rangle
$$

and we consider two cases.

Case 1: $x_{0}^{* *} \in X$.

Then (3) follows directly from Fact 2.1.

Case 2: $x_{0}^{* *} \in X^{* *} \backslash X$.

By Fact 1.2 , there exists a bounded net $\left(x_{\alpha}\right)_{\alpha \in I}$ in $X$ that weak* converges to $x_{0}^{* *}$. Thus, we have

$$
M=\sup _{\alpha \in I}\left\|x_{\alpha}\right\|<+\infty
$$

and

$$
\left\langle x_{\alpha}, x_{0}^{*}\right\rangle \rightarrow\left\langle x_{0}^{* *}, x_{0}^{*}\right\rangle
$$

Now we consider two subcases.

Subcase 2.1: There exists $\alpha \in I$, such that $\left(x_{\alpha}, x_{0}^{*}\right) \in \operatorname{gra} A$.

By definition,

$$
F_{A}\left(x_{0}^{* *}, x_{0}^{*}\right) \geq\left\langle x_{\alpha}, x_{0}^{*}\right\rangle+\left\langle x_{0}^{* *}, x_{0}^{*}\right\rangle-\left\langle x_{\alpha}, x_{0}^{*}\right\rangle=\left\langle x_{0}^{* *}, x_{0}^{*}\right\rangle .
$$


Hence (3) holds.

Subcase 2.2: We have

$$
\left(x_{\alpha}, x_{0}^{*}\right) \notin \operatorname{gra} A, \quad \forall \alpha \in I .
$$

Set

$$
U_{\varepsilon}=\left[0, x_{0}^{*}\right]+\varepsilon U_{X^{*}},
$$

where $\varepsilon>0$. Observe that $U_{\varepsilon}$ is open and convex. Since $(0,0) \in$ gra $A$, we have, by definition of $U_{\varepsilon}, 0 \in \operatorname{ran} A \cap U_{\varepsilon}$ and $x_{0}^{*} \in U_{\varepsilon}$. In view of (6) and because $A$ is of type (FP), there exists a net $\left(a_{\alpha, \varepsilon}, a_{\alpha, \varepsilon}^{*}\right)$ in gra $A$ such that $a_{\alpha, \varepsilon}^{*} \in U_{\varepsilon}$ and

$$
\left\langle a_{\alpha, \varepsilon}, x_{0}^{*}\right\rangle+\left\langle x_{\alpha}, a_{\alpha, \varepsilon}^{*}\right\rangle-\left\langle a_{\alpha, \varepsilon}, a_{\alpha, \varepsilon}^{*}\right\rangle>\left\langle x_{\alpha}, x_{0}^{*}\right\rangle, \quad \forall \alpha \in I .
$$

Now fix $\alpha \in I$. By (요),

$$
\left\langle a_{\alpha, \varepsilon}, x_{0}^{*}\right\rangle+\left\langle x_{0}^{* *}, a_{\alpha, \varepsilon}^{*}\right\rangle-\left\langle a_{\alpha, \varepsilon}, a_{\alpha, \varepsilon}^{*}\right\rangle>\left\langle x_{0}^{* *}-x_{\alpha}, a_{\alpha, \varepsilon}^{*}\right\rangle+\left\langle x_{\alpha}, x_{0}^{*}\right\rangle .
$$

Hence,

$$
F_{A}\left(x_{0}^{* *}, x_{0}^{*}\right)>\left\langle x_{0}^{* *}-x_{\alpha}, a_{\alpha, \varepsilon}^{*}\right\rangle+\left\langle x_{\alpha}, x_{0}^{*}\right\rangle .
$$

Since $a_{\alpha, \varepsilon}^{*} \in U_{\varepsilon}$, there exist

$$
t_{\alpha, \varepsilon} \in[0,1] \text { and } b_{\alpha, \varepsilon}^{*} \in U_{X^{*}}
$$

such that

$$
a_{\alpha, \varepsilon}^{*}=t_{\alpha, \varepsilon} x_{0}^{*}+\varepsilon b_{\alpha, \varepsilon}^{*} .
$$

Using (9), (11), and (4), we deduce that

$$
\begin{aligned}
F_{A}\left(x_{0}^{* *}, x_{0}^{*}\right) & >\left\langle x_{0}^{* *}-x_{\alpha}, t_{\alpha, \varepsilon} x_{0}^{*}+\varepsilon b_{\alpha, \varepsilon}^{*}\right\rangle+\left\langle x_{\alpha}, x_{0}^{*}\right\rangle \\
& =t_{\alpha, \varepsilon}\left\langle x_{0}^{* *}-x_{\alpha}, x_{0}^{*}\right\rangle+\varepsilon\left\langle x_{0}^{* *}-x_{\alpha}, b_{\alpha, \varepsilon}^{*}\right\rangle+\left\langle x_{\alpha}, x_{0}^{*}\right\rangle \\
& \geq t_{\alpha, \varepsilon}\left\langle x_{0}^{* *}-x_{\alpha}, x_{0}^{*}\right\rangle-\varepsilon\left\|x_{0}^{* *}-x_{\alpha}\right\|+\left\langle x_{\alpha}, x_{0}^{*}\right\rangle \\
& \geq t_{\alpha, \varepsilon}\left\langle x_{0}^{* *}-x_{\alpha}, x_{0}^{*}\right\rangle-\varepsilon\left(\left\|x_{0}^{* *}\right\|+M\right)+\left\langle x_{\alpha}, x_{0}^{*}\right\rangle .
\end{aligned}
$$

In view of (10) and since $\alpha \in I$ was chosen arbitrarily, we take the limit in (12) and obtain with the help of (5) that

$$
F_{A}\left(x_{0}^{* *}, x_{0}^{*}\right) \geq-\varepsilon\left(\left\|x_{0}^{* *}\right\|+M\right)+\left\langle x_{0}^{* *}, x_{0}^{*}\right\rangle .
$$

Next, letting $\varepsilon \rightarrow 0$ in (13), we have

$$
F_{A}\left(x_{0}^{* *}, x_{0}^{*}\right) \geq\left\langle x_{0}^{* *}, x_{0}^{*}\right\rangle .
$$

Therefore, (3) holds in all cases.

We now obtain the promised corollary: 
Corollary 3.2 Let $A: X \rightrightarrows X^{*}$ be maximally monotone. Then the following are equivalent.

(i) A is of type (D).

(ii) A is of type (NI).

(iii) $A$ is of type (FP).

Proof. First (i) implies (iii) is Fact 2.2, next Theorem 3.1 shows (iii) implies (ii); while Fact 2.3 implies concludes the circle with (ii) implies (i).

We note that while the result is now quite easy, it remained inaccessible until [12, Theorem 4.4] was available.

Remark 3.3 Let $A: X \rightrightarrows X^{*}$ be maximally monotone. Corollary 3.2 establishes the equivalences of the key types (D), (NI), and (FP), which as noted all hold when $X$ is reflexive or $A=\partial f$, where $f: X \rightarrow]-\infty,+\infty]$ is convex, lower semicontinuous, and proper (see [6, 17, 19]).

Furthermore, these notions are also equivalent to type (ED), see [20. For a nonlinear operator they also coincide with uniqueness of maximal extensions to $X^{* *}$ (see [12]). In [6, p. 454] there is discussion of this result and of the linear case.

Finally, when $A$ is a linear relation, it has recently been established that all these notions coincide with monotonicity of the adjoint multifunction $A^{*}$ (see [1]).

\section{Acknowledgments}

Heinz Bauschke was partially supported by the Natural Sciences and Engineering Research Council of Canada and by the Canada Research Chair Program. Jonathan Borwein was partially supported by the Australian Research Council. Xianfu Wang was partially supported by the Natural Sciences and Engineering Research Council of Canada.

\section{References}

[1] H.H. Bauschke, J.M. Borwein, X. Wang and L. Yao, "For maximally monotone linear relations, dense type, negative-infimum type, and Fitzpatrick-Phelps type all coincide with monotonicity of the adjoint", submitted; http://arxiv.org/abs/1103.6239v1, March 2011. 
[2] H.H. Bauschke and P.L. Combettes, Convex Analysis and Monotone Operator Theory in Hilbert Spaces, Springer-Verlag, 2011.

[3] J.M. Borwein, "Maximal monotonicity via convex analysis", Journal of Convex Analysis, vol. 13, pp. 561-586, 2006.

[4] J.M. Borwein, "Maximality of sums of two maximal monotone operators in general Banach space", Proceedings of the American Mathematical Society, vol. 135, pp. 3917-3924, 2007.

[5] J.M. Borwein, "Fifty years of maximal monotonicity", Optimization Letters, vol. 4, pp. 473490, 2010.

[6] J.M. Borwein and J.D. Vanderwerff, Convex Functions, Cambridge University Press, 2010.

[7] R.S. Burachik and A.N. Iusem, Set-Valued Mappings and Enlargements of Monotone Operators, Springer-Verlag, 2008.

[8] M. Fabian, P. Habala, P. Hájek, V. Montesinos Santalucía, J. Pelant and V. Zizler, Functional Analysis and Infinite-Dimensional Geometry, Springer-Verlag, 2001.

[9] S. Fitzpatrick, "Representing monotone operators by convex functions", in Workshop/Miniconference on Functional Analysis and Optimization (Canberra 1988), Proceedings of the Centre for Mathematical Analysis, Australian National University, vol. 20, Canberra, Australia, pp. 59-65, 1988.

[10] S. Fitzpatrick and R.R. Phelps, "Bounded approximants to monotone operators on Banach spaces", Annales de l'Institut Henri Poincaré. Analyse Non Linéaire, vol. 9, pp. 573-595, 1992.

[11] J.-P. Gossez, "Opérateurs monotones non linéaires dans les espaces de Banach non réflexifs", Journal of Mathematical Analysis and Applications, vol. 34, pp. 371-395, 1971.

[12] M. Marques Alves and B.F. Svaiter, "On Gossez type (D) maximal monotone operators", Journal of Convex Analysis, vol. 17, pp. 1077-1088, 2010.

[13] R.E. Megginson, An Introduction to Banach Space Theory, Springer-Verlag, 1998.

[14] R.R. Phelps, Convex Functions, Monotone Operators and Differentiability, 2nd Edition, Springer-Verlag, 1993.

[15] R.T. Rockafellar and R.J-B Wets, Variational Analysis, 3rd Printing, Springer-Verlag, 2009.

[16] S. Simons, "The range of a monotone operator", Journal of Mathematical Analysis and Applications, vol. 199, pp. 176-201, 1996.

[17] S. Simons, Minimax and Monotonicity, Springer-Verlag, 1998.

[18] S. Simons, "Five kinds of maximal monotonicity", Set-Valued and Variational Analysis, vol. 9, pp. 391-409, 2001. 
[19] S. Simons, From Hahn-Banach to Monotonicity, Springer-Verlag, 2008.

[20] S. Simons, "Banach SSD Spaces and classes of monotone sets", Journal of Convex Analysis, vol. 18, pp. 227-258, 2011.

[21] C. Zălinescu, Convex Analysis in General Vector Spaces, World Scientific Publishing, 2002. 